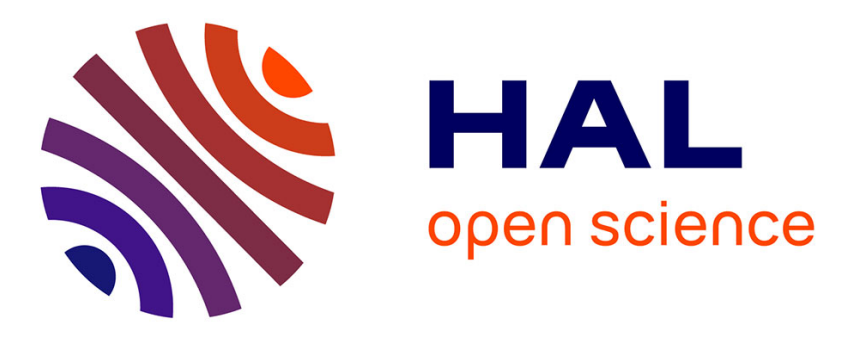

\title{
Statistical study of blood cell populations by very wide-field bimodal phase/ fluorescence imaging
}

Isaure Le Cardinal de Kernier, Stéphanie Bressieux, Nelly Rongeat, Anais Ali-Cherif, Sophie Morales, Serge Monneret, Pierre Blandin

\section{- To cite this version:}

Isaure Le Cardinal de Kernier, Stéphanie Bressieux, Nelly Rongeat, Anais Ali-Cherif, Sophie Morales, et al.. Statistical study of blood cell populations by very wide-field bimodal phase/ fluorescence imaging. Optical Diagnostics and Sensing XIX: Toward Point-of-Care Diagnostics, Feb 2019, San Francisco, United States. pp.10, 10.1117/12.2506531 . hal-02473883

\section{HAL Id: hal-02473883 \\ https://hal.science/hal-02473883}

Submitted on 11 Feb 2020

HAL is a multi-disciplinary open access archive for the deposit and dissemination of scientific research documents, whether they are published or not. The documents may come from teaching and research institutions in France or abroad, or from public or private research centers.
L'archive ouverte pluridisciplinaire HAL, est destinée au dépôt et à la diffusion de documents scientifiques de niveau recherche, publiés ou non, émanant des établissements d'enseignement et de recherche français ou étrangers, des laboratoires publics ou privés. 


\title{
Statistical study of blood cells population by very wide-field bimodal phase/ fluorescence imaging
}

\author{
Isaure de Kernier ${ }^{\mathrm{a}, \mathrm{b}, *}$, Stéphanie Bressieux ${ }^{\mathrm{c}}$, Nelly Rongeat ${ }^{\mathrm{c}}$, Anaïs Ali-Cherif ${ }^{\mathrm{c}}$, Sophie Morales ${ }^{\mathrm{a}}$, \\ Serge Monneret ${ }^{\mathrm{b}}$, Pierre Blandin ${ }^{\mathrm{a}}$ \\ ${ }^{a}$ Univ. Grenoble Alpes, F-38000 Grenoble, France, CEA, LETI, MINATEC Campus, Technologies \\ for Healthcare and Biology division, F-38054 Grenoble, France; ${ }^{\mathrm{b}}$ Aix Marseille Univ, CNRS, \\ Centrale Marseille, Institut Fresnel, Marseille, France; ${ }^{c}$ HORIBA ABX SAS, Montpellier, France
}

\begin{abstract}
Very wide-field of view imaging can provide statistical data on large cell populations in a single acquisition. It can be used as an alternative to standard fluorescence flow cytometry methods for applications in hematology. We will describe a multimodal imaging system combining brightfield, phase and fluorescence contrasts. Its greater simplicity and lower cost compared to flow cytometry make it suitable for Point-Of-Care applications. The system's resolution was characterized on calibrated beads and targets. We illustrate the potential of the single-shot imaging approach in hematology by showing classification of white blood cells morphologies.
\end{abstract}

Keywords: optics, statistics, fluorescence, phase, in-line holography, microscopy

\section{INTRODUCTION}

The study of cellular populations requires acquiring information on a significant number of cells to ensure statistically reliable results. In imaging flow cytometry, the statistics comes from the flow of objects in front of a high magnification imaging system. Such a solution typically ensures a throughput of $500-1000$ cells per second ${ }^{1}$. This is much lower than the statistics achieved using flow cytometry, that tops 5000 cells per seconds ${ }^{2}$. This affects the period of time over which the acquisition is done. Recent research work have examined the method and applications of very-wide field imaging. If very large objects such as embryos ${ }^{3}$ or large numbers of small objects ${ }^{4}$ such as blood cells need to be studied, then acquiring a large Field Of View (FOV) in a single-shot minimizes the total number of images required. The figure below illustrates that the FOV of a standard microscope with a 10x objective is 100 times smaller than that of a 1x objective.

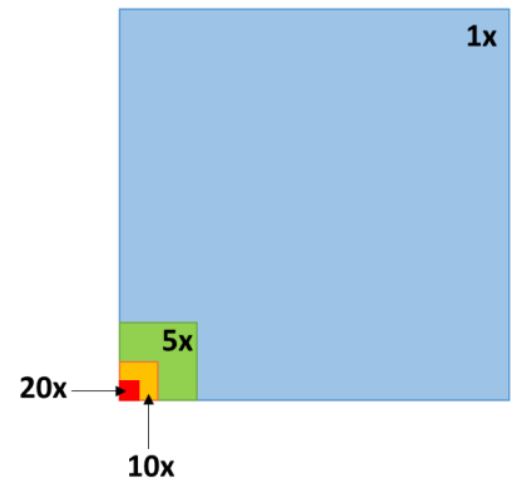

Figure 1 - Theoretical comparison of the FOV of objectives depending on their magnification.

In this work we aim at developing a high throughput single-shot imaging system. Such a system enables statistical studies of cell populations without requiring any flow of cells as in imaging flow cytometry or scanning as in laser- 
scanning cytometry ${ }^{5}$. We demonstrate that we are able to acquire transmission, phase and fluorescence images of a 30 $\mathrm{mm}^{2}$ FOV. Furthermore, we illustrate the potential of our system for statistical analysis of blood cell populations. The morphologies of labeled nuclei of purified White Blood Cells (WBC) sub-types are considered in fluorescence. This proof of concept experiment suggests that statistical analysis of blood cell populations is possible.

\section{DESIGN OF AN IN-LINE IMAGING SYSTEM}

Combining contrasts in an imaging systems requires registration of the images from each modality. We chose to design a system with a single imaging pathway in order to obtain hardware registrated images, such that no additional image postprocessing is necessary to merge information. The common imaging path set up with a single objective (referred to as Macro Lens in the figure) and a CMOS sensor is illustrated in Figure 2. Advantageously this solution is cost-effective.

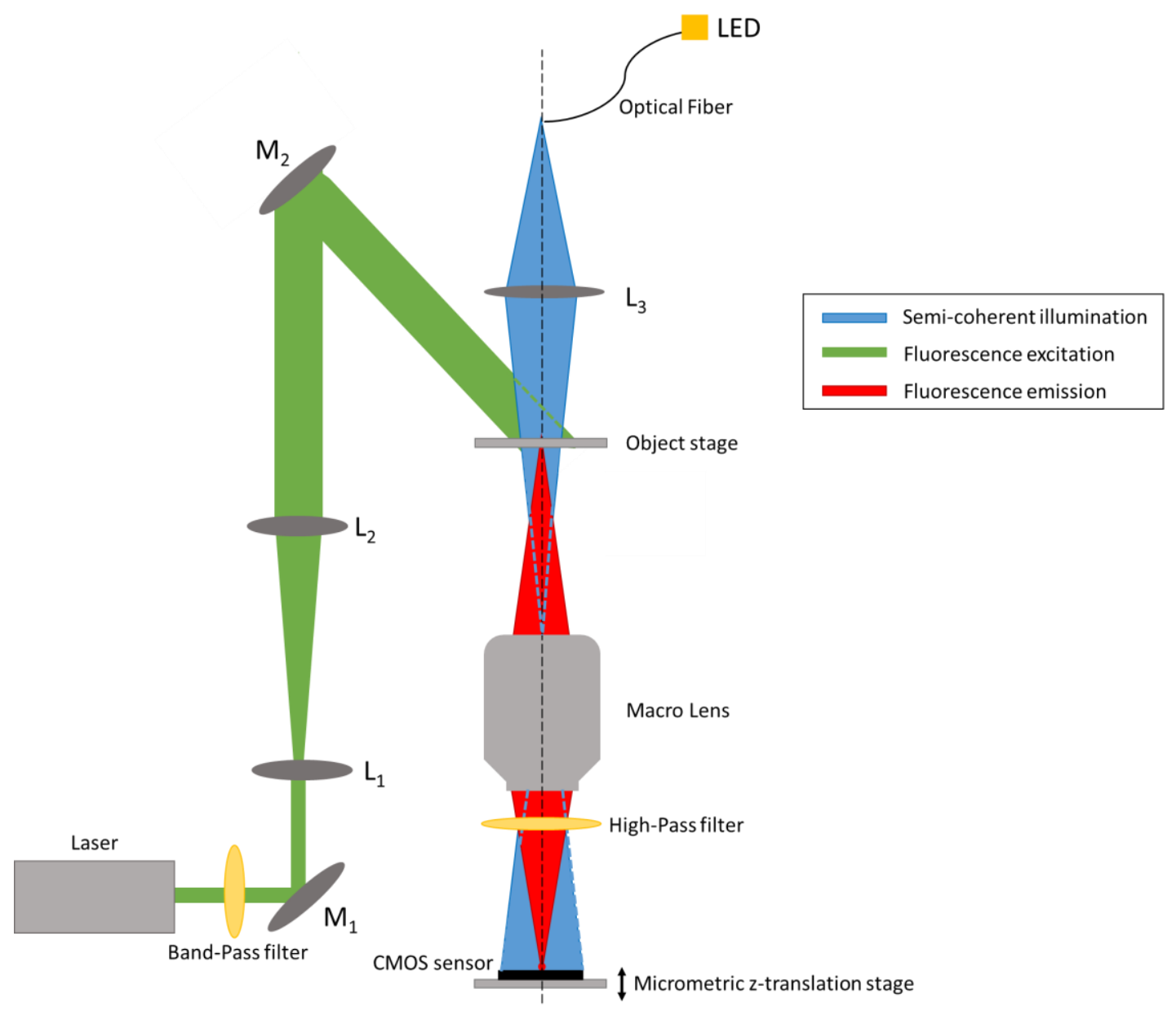

Figure 2 - Wide FOV multi-modal imaging system

The aim of the system is to perform single-shot statistical imaging of micrometric objects. This implies that both FOV and resolution should be optimized. To obtain a large FOV, we chose a SRL camera lens from macrophotography series of Canon Inc. (Canon EF $100 \mathrm{~mm}$ f/2.8 Macro USM, Canon Inc., USA). The numerical aperture of this lens is measured to be $0.113 \pm 0.003$. The PSF diameter is theoretically determined to be $D=\frac{0.61 \lambda}{N A} \sim 2.81 \mu \mathrm{m}$ at $520 \mathrm{~nm}$. Hence, according to the Shannon-Nyquist criteria, the optimal pixel pitch is $1.4 \mu \mathrm{m}$. However, as the 
development of sensors has been driven by low-cost high resolution cameras for phones, small pixel pitch down to 1.1 $\mu \mathrm{m}$ are available but at the detriment of the chip size. For our system, we make the compromise of choosing a monochromatic CMOS $11 \mathrm{Mpx} 6.4$ by $4.6 \mathrm{~mm}^{2}$ sensor with a $1.67 \mu \mathrm{m}$ pixel pitch (UI-1492LE-M, IDS GmbH, Germany) resulting in a $29.4 \mathrm{~mm}^{2}$ FOV. This solution is found to be a good resolution-FOV compromise. However, a larger chip with smaller pixels would enhance the performances of the set-up.

The chosen macro lens is designed to achieve a 1:1 magnification ratio. In this configuration, the object plane is $145 \mathrm{~mm}$ away from the front lens of the objective. The sensor is mounted on a manual micrometric z-axis translation stage (SM1Z, Thorlabs Inc., USA) with a $25 \mathrm{~mm}$ range to enable precise positioning of the image plane.

Brightfield images can be acquired by illuminating the object in an in-line configuration, as illustrated in Figure 2. The illumination module comprises a $520 \mathrm{~nm}$ low-cost LED (XLamp® XM-L ${ }^{\mathrm{TM}}$ Color LED, Cree Inc., USA) coupled into an optical fiber (FG200UEA, Thorlabs Inc., USA) and shaped into a convergent beam by an additional lens (LAT075, Thorlabs Inc., USA).

Several methods enable to obtain a phase contrast from out-of-focus brightfield images. With the specifications mentioned, our system's brightfield illumination is coherent enough to enable in-line holography ${ }^{6}$ acquisitions. State of the art algorithms ${ }^{7}$ are used to reconstruct an amplitude and a phase map from the acquired hologram.

Both brightfield and phase imaging allows to obtain morphological information on the studied object. However, this is not sufficient to discriminate sub-types of cells whose morphology is similar. For this reason, it is interesting to introduce a fluorescence contrast that is more specific. In this work, we chose to work with the Thiazole Orange fluorescence dye ${ }^{8}$. The excitation and emission specifications for this dye are given in Figure 3. A laser (LBX-505, Oxxius, France) was combined to a bandpass excitation filter (FF01-504/12, Semrock, USA) and used as the excitation source. The excitation beam was tilted with an angle $\sim 45^{\circ}$ from the optical axis to avoid direct excitation to be incident on the sensor, as illustrated in Figure 2. This reduced the constraints on the emission filter. The emission filter (FF01536/40-25) was added in the imaging path between the objective and the sensor to filter out the excitation wavelength.

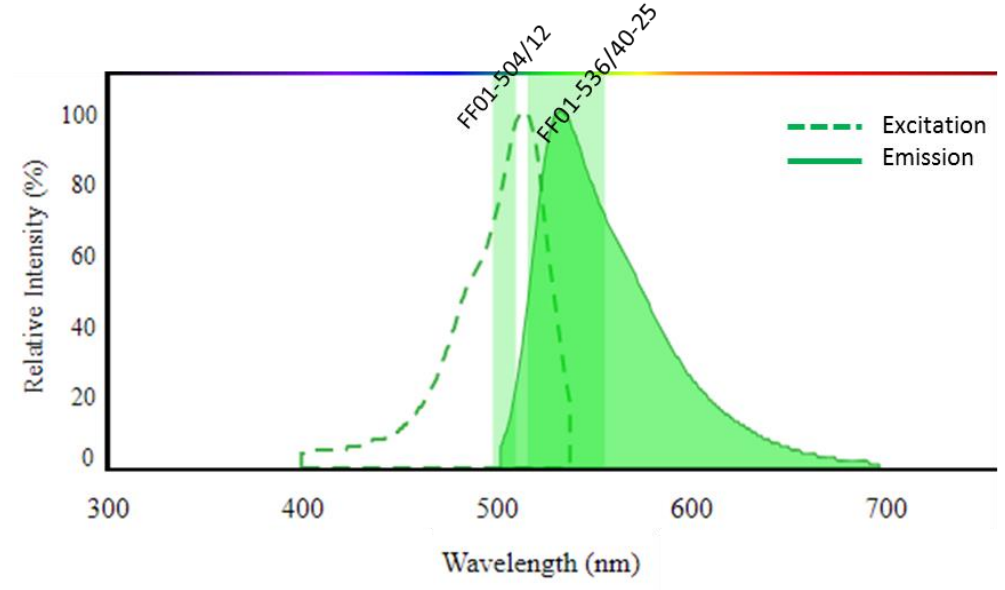

Figure 3 - Excitation and emission spectra of Thiazole Orange dye. The pass-band filters used for excitation and emission are also shown.

Because the brightfield illumination wavelength was chosen higher than the filter's cut-off wavelength, brightfield and holographic imaging could be performed without removing the fluorescence emission filter. 


\section{CHARACTERIZATION OF THE SYSTEM}

The system combines a very wide field of view of $29.4 \mathrm{~mm}^{2}$ with a micrometric resolution, as illustrated in Figure 4 . To characterize the resolution, we imaged an amplitude 1951 USAF resolution test chart. Here resolution is defined as the width of the last group of bars whose contrast is over 10\%. Under blue LED illumination, we measured a resolution of $2.76 \pm 0.15 \mu \mathrm{m}$ in the center of the FOV.
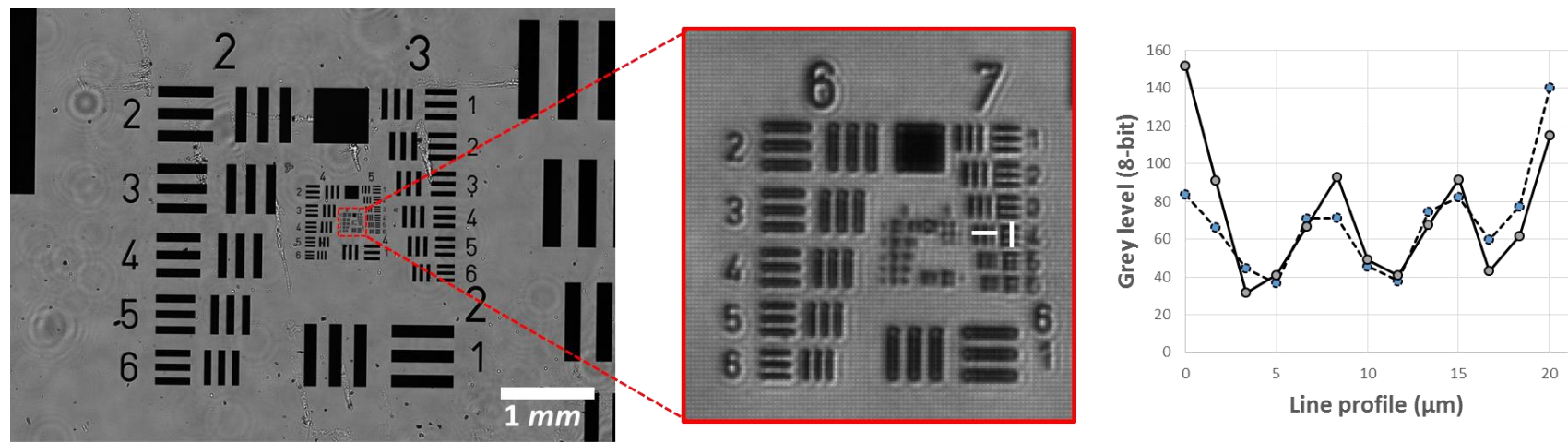

Figure 4 - USAF resolution target illustrating the wide FOV and the resolution.

Furthermore, the fluorescence resolution was experimentally determined. A solution of $1 \mu \mathrm{m}$ GFP-fluorescent beads was evaporated on a standard microscope glass slide. In the acquired fluorescence image, individual beads resulted in Point Spread Functions (PSF). Resolution was defined as the FWHM of the experimental discrete data points of a line profile of a PSF. An average resolution of $3.72 \pm 0.25 \mu \mathrm{m}$ was obtained in the center of the FOV, as illustrated in Figure 5.
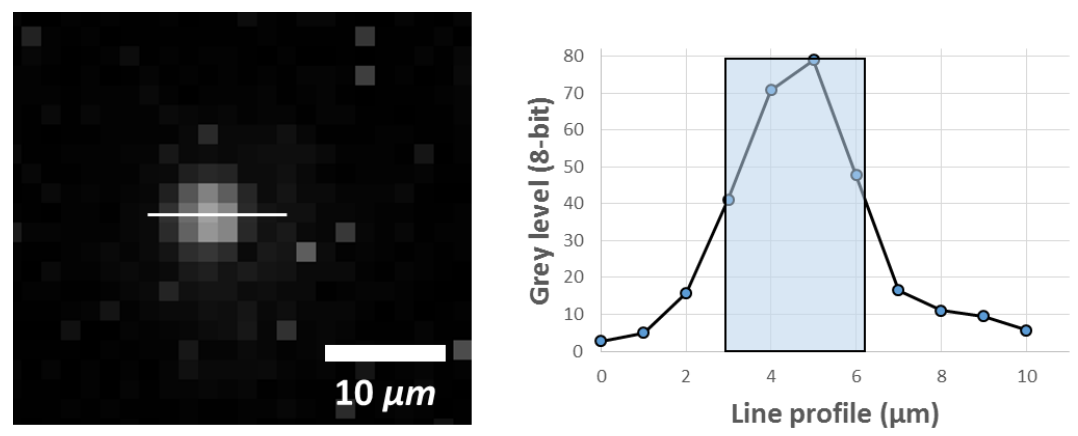

Figure 5 - PSF. Image of a fluorescent GFP - $1 \mu \mathrm{m}$ bead. Line profile pointing out the FWHM.

These experimental evaluations of the multi-modal resolution indicate that resolved imaging of small biological objects should be possible.

\section{STATISTICAL STUDY OF WHITE BLOOD CELL MORPHOLOGIES}

The classification of the three sub-populations of White Blood Cells (WBC) is an important basic information, usually provided in routine blood tests. WBC can be separated into three distinct populations: monocytes, lymphocytes and granulocytes. The proportion of these different types of blood cells is used for medical diagnosis, such as Dengue fever ${ }^{9,10}$. 
To demonstrate the imaging capacity of our system, we acquired bi-modal images of blood smears of WBC purified populations. We show that the fluorescence image can be used to discriminate the sub-types of WBC.

The study was achieved on three whole blood samples. For each $2 \mathrm{~mL}$ whole blood sample, a specific sub-population of WBC was purified. Purification of lymphocyte was done using the RosetteSep Human total Lymphocyte enrichment kit (15223, StemCell). The purified solution was diluted in $500 \mu \mathrm{L}$ of PBS $+2 \%$ FBS (07905, StemCell, USA). Purification of granulocytes was done using the EasySep Direct Human Pan-Granulocytes Isolation kit (19659, StemCell, USA). Finally, purification of monocytes was done in two steps. First the platelets were disposed of by three consecutive centrifugations for $15 \mathrm{~min}$ at 200g. After each centrifugation the supernatant was eliminated and $2 \mathrm{~mL}$ PBS $+2 \%$ FBS and $1 \mathrm{mM}$ EDTA (ED2SS, Sigma, USA) were added. Then, the monocytes were purified using the RosetteSep Human Monocyte Enrichment Cocktail kit (15028, StemCell, USA). The purified fraction was diluted in 500 $\mu \mathrm{L}$ of PBS $+2 \%$ FBS and 1 mM EDTA. The ABX Pentra DX 120 (Horiba Medical, Japan) hematology analyzer was used to verify the purification process. We established that the sub-population were purified with a rate above $98 \%$ for granulocytes, $95 \%$ for lymphocytes and $74 \%$ for monocytes.

Then, the purified WBC samples were specifically labeled with Thiazole Orange dye (390063, Sigma-Aldrich Inc., USA). This nucleic acids marker is known to have a high quantum yield ${ }^{8}$. A marking solution was obtained by dissolving at $1 \mathrm{mg} / \mathrm{mL}$ Thiazole Orange in methanol, and by then diluting it to $18 \mu \mathrm{g} / \mathrm{mL}$ in PBS (D8537, Sigma-Aldrich Inc., USA). The samples were incubated for 30 minutes with $1 \mathrm{~mL}$ of the marking solution.

Finally, blood smears were made on strandard microscope slides using the automated blood smearing device Hemaprep (Horiba Medical, Japan).

For a healthy person, WBC account for roughly $0.1 \%$ of the blood cells. The wide field of view of the systems allowed to detect a large number of labelled cells. In this experiment, we acquired sequentially a brightfield and a fluorescence image. As pointed out previously, the bimodal images required no registration thanks to the common imaging path. On a single image, we were able to detect 1000 - $3000 \mathrm{WBC}$, as illustrated in Figure 6.

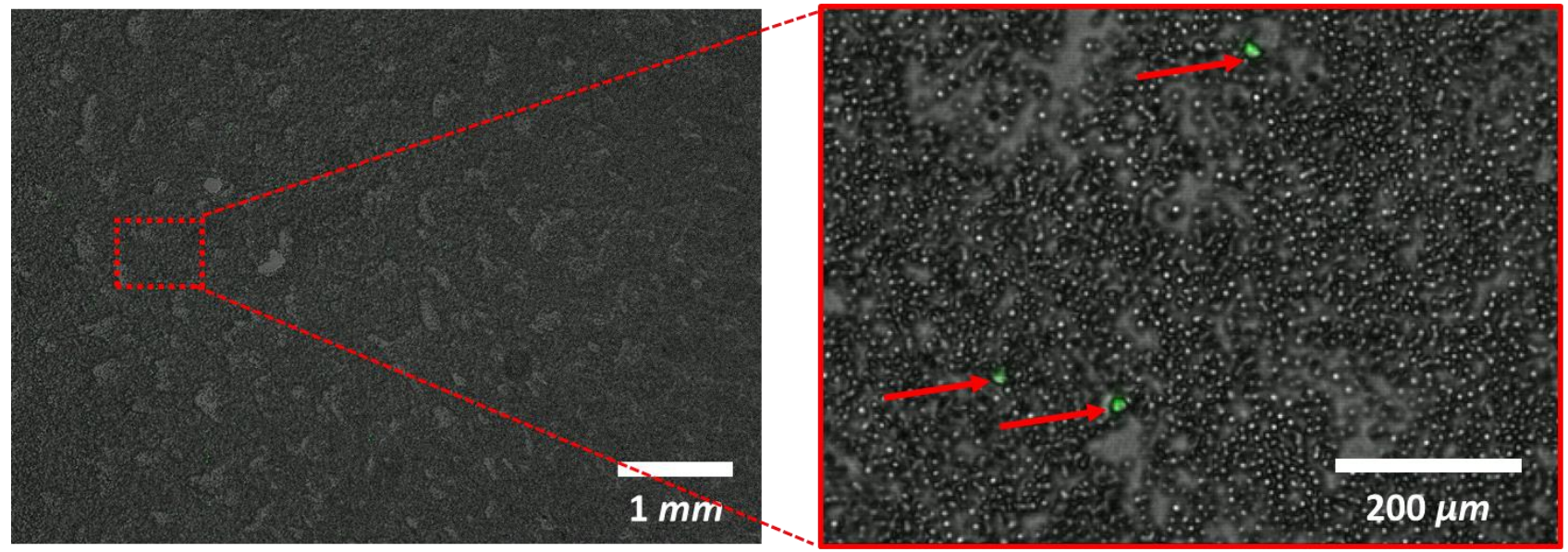

Figure 6 - Superposed transmission (in grey scale) and fluorescent (artificial green color) images of labelled WBC.

To demonstrate the performance of our system, we compared the fluorescence images from our set up to those acquired using a standard microscope (BX51, Olympus, USA) with a 40x objective. In particular, the morphology of the nuclei of WBC were examined. Figure 7 shows similar morphologies of WBC nuclei imaged using the two systems. Although the images were acquired on the same slide and on the same day, it is not certain that the exact same cells were imaged. The registration of the images from the reference microscope and our system is difficult given the size of the FOVs. 
Granulocytes are the most common WBC type (40 to $75 \%$ ), they are roughly $12 \mu \mathrm{m}$ in diameter and their nucleus has a characteristic multi-lobe or irregular-shape. As illustrated on a bi-lobes nucleus in Figure 7, our system's resolution was sufficient to distinguish the morphological features of granulocytes. Lymphocytes account for 20 to $40 \%$ of $\mathrm{WBC}$, are regular and round-shaped cells of $7 \mu \mathrm{m}$ diameter with a nucleus occupying almost the entire cell. Monocytes represent 2 to $10 \%$ of $\mathrm{WBC}$, are large $15-20 \mu \mathrm{m}$ cells with a granular horse shoe shaped nucleus. As illustrated in Figure 7, lymphocytes and monocytes could be differentiated. The size difference of the nuclei of two subtypes were discernable, and the fluorescence signal of monocytes were more diffuse.
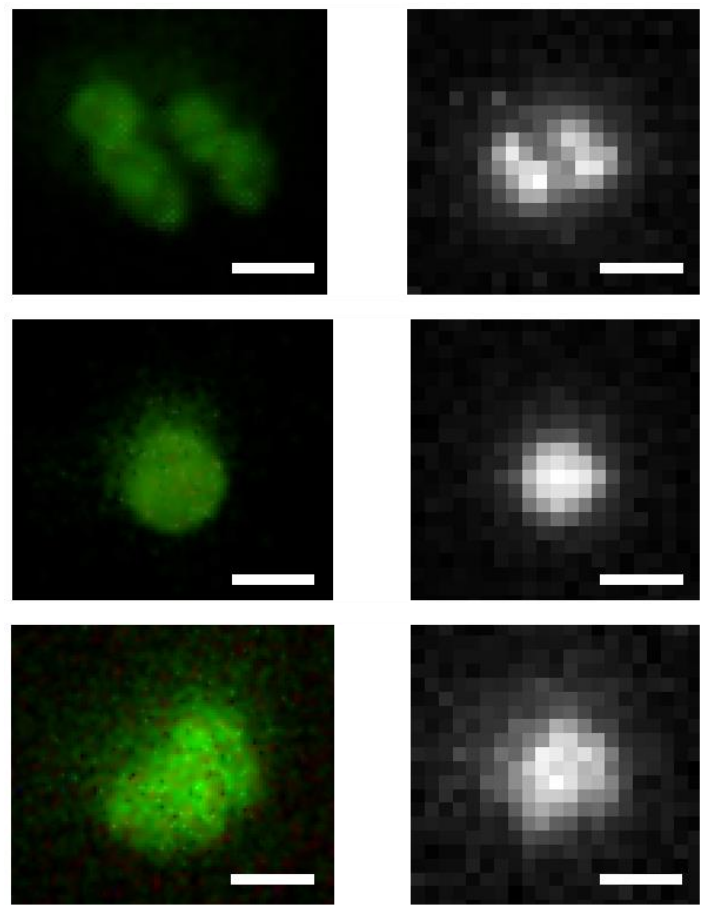

Figure 7 Acquisitions of the fluorescence of granulocytes, lymphocytes and monocytes (respectively) with a standard epifluorescence microscope with a 40x objective and with our system. The field of views are cropped. Scale bar is $10 \mu \mathrm{m}$.

These images indicate that our system has a sufficient resolution to point out the key features of each WBC sub-type. Thus, these results suggest that it might be possible to perform statistical determination of the ratios of WBC populations within a whole blood sample.

\section{CONCLUSION}

We described an imaging system combining in-line holography, brightfield and fluorescence over a $30 \mathrm{~mm}^{2} \mathrm{FOV}$, achieving a 3-4 $\mu \mathrm{m}$ resolution. This system takes advantage of a SLR camera lens with a front lens wider than those usually used in microscopy. Such a system can target many biological applications. To illustrate its potential, we chose to show its performances in imaging WBC sub-types. Results suggest that the system does combine a resolution sufficient to distinguish the morphological features of the nuclei, while the FOV is sufficiently large to be able to infer statistically relevant information. The next step of this work would be to image a blood smear of a whole i.e. not purified blood sample and show that it is possible to classify the detected WBC into distinct populations. 


\section{REFERENCES}

[1] Zuba-Surma, E. K., Kucia, M., Abdel-Latif, A., Jr, J. W. L. and Ratajczak, M. Z., "The ImageStream System: a key step to a new era in imaging," 12.

[2] Adan, A., Alizada, G., Kiraz, Y., Baran, Y. and Nalbant, A., "Flow cytometry: basic principles and applications," Critical Reviews in Biotechnology 37(2), 163-176 (2017).

[3] McConnell, G., Trägardh, J., Amor, R., Dempster, J., Reid, E. and Amos, W. B., "A novel optical microscope for imaging large embryos and tissue volumes with sub-cellular resolution throughout," eLife 5, e18659 (2016).

[4] Hatch, A. C., Fisher, J. S., Tovar, A. R., Hsieh, A. T., Lin, R., Pentoney, S. L., Yang, D. L. and Lee, A. P., "1-Million droplet array with wide-field fluorescence imaging for digital PCR," Lab on a Chip 11(22), 3838 (2011).

[5] Darzynkiewicz, Z., Bedner, E., Li, X., Gorczyca, W. and Melamed, M. R., "Laser-Scanning Cytometry: A New Instrumentation with Many Applications," Experimental Cell Research 249(1), 1-12 (1999).

[6] Singh, D. K., Ahrens, C. C., Li, W. and Vanapalli, S. A., "Label-free, high-throughput holographic screening and enumeration of tumor cells in blood," Lab on a Chip 17(17), 2920-2932 (2017).

[7] Hervé, L. and Allier, C. "Method for observing a sample, by calculation of a complex image, Patent $\mathrm{n}^{\circ} \mathrm{WO} / 2017 / 162985 "(2017)$.

[8] Lee, L. G., Chen, C.-H. and Chiu, L. A., "Thiazole orange: A new dye for reticulocyte analysis," Cytometry 7(6), 508-517 (1986).

[9] Forcucci, A., Pawlowski, M. E., Majors, C., Richards-Kortum, R. and Tkaczyk, T. S., "All-plastic, miniature, digital fluorescence microscope for three part white blood cell differential measurements at the point of care," Biomedical Optics Express 6(11), 4433 (2015).

[10] Buttarello, M. and Plebani, M., "Automated Blood Cell Counts: State of the Art," American Journal of Clinical Pathology 130(1), 104-116 (2008). 